\title{
Numerical and Experimental Investigations of Coupling Effects in Anisotropic Elastic Rotors*
}

\author{
HORST IRRETIER $^{a}$, GEORGES JACQUET-RICHARDET ${ }^{\mathrm{b}, \dagger}$ and FRANK REUTER ${ }^{\mathrm{a}}$ \\ anstitute of Mechanics, University of Kassel, D-34109 Kassel, Germany; ' ${ }^{\mathrm{b}}$ Laboratoire de Mécanique des Structures, \\ INSA' de Lyon, F-69100 Villeurbanne, France
}

(Received in final form 7 July 1998)

\begin{abstract}
It is known that in elastic disc-shaft systems in particular, the one-nodal-diameter mode of the discs can be highly coupled with the bending modes of the shaft. Consequently, when the system rotates, the elastic modes of the flexible discs are coupled with the gyroscopic modes of the flexible shaft equipped with rigid discs. In the paper this coupling effect is investigated numerically and experimentally.

A numerical model, based on a finite element cyclic symmetry approach, is presented. This model has been developed for studying the wheel-shaft coupling effects on the global behavior of turbomachinery rotors. In order to better illustrate the phenomenon involved and to validate the model, the method is applied here to a thin tuned and detuned circular disc mounted on an elastic shaft. Related frequency and mode shapes of the rotating assembly are discussed. Additional experimental results, based on an experimental modal analysis technique for rotating structures, are presented. Both numerical and experimental results are compared.
\end{abstract}

Keywords: Rotordynamics, Disc-shaft coupling, Natural frequencies, Campbell diagram

\section{INTRODUCTION}

Traditionally, the analysis of the dynamic behavior of rotating disc-shaft systems is performed using two different approaches. The rotordynamic approach is focused on shaft behavior and assumes that the effect of wheel flexibility is negligible. On the other hand, the bladed-disc approach assumes that the shaft is rigid and analyses the behavior of the different wheels separately. These approaches are usually efficient and accurate enough. However, there is an increasing number of cases where coupling effects between bladed-disks and shafts cannot be ignored. Possible couplings have been pointed out in early studies by Dopkin and Shoup (1974), Chivers and Nelson (1975), Klompas (1978), and are now better understood owing to the development of various analytical analyses: Palladino and Rossettos (1982), Brémand et al. (1986), Crawley et al. (1983, 1986), Loewy and

\footnotetext{
* This paper was originally presented at ISROMAC-7.

${ }^{\dagger}$ Corresponding author.
} 
Khader (1984), Khader et al. (1990, 1991), Wu and Flowers (1992). Particular features such as inertial coupling, mistuning influence and forced response behavior have been examined. Even recently, an analytical procedure has been developed by Chun and Lee (1996) in order to study the influence of rotation, blade stagger and blade pretwist on coupled behaviors.

These analytical approaches, while very useful, are based on simplified formulations and thus cannot be applied efficiently to real structures with complex geometries. On the contrary, direct and precise finite element modeling of the whole coupled system would be attractive, but cannot be performed due to the unrealistic computer resources needed. Consequently, intermediate modeling techniques have to be proposed and assessed. A first type of intermediate model consists in analysing the shaft with rigid disc and the bladed assemblies separately, and then solving the coupled problem after a modal reduction based on the calculated mode shapes (Zhang et al., 1994). This model should give quite accurate results when the link between the shaft and the disc is rigid, but it is believed to be too restrictive for general applications. Dealing with the whole coupled assembly, direct modeling is possible using an axisymmetrical description of the rotor (Géradin and Kill, 1984; Stephenson and Rouch, 1993). The resulting model should be effective in certain cases but it is penalised by the axisymmetrical hypothesis and by the modeling difficulties associated. Finally, it appears that the most interesting models are those based on the cyclic symmetrical properties of the structure (Géradin and Kill, 1986; Hohlrieder et al., 1994; 1997; Jacquet-Richardet et al., 1996). However, these models remain heavy and, in this case, special procedures should be developed in order to maintain the computer requirements at an acceptable level. The formulation presented here is based on a global analysis of rotating assemblies modeled using a finite element technique. The undamped non-rotating system is first analysed using the wave propagation method associated with a component mode reduction. Then, the whole flexible system, submitted to centrifugal and gyroscopic effects, is analysed after a modal reduction.

In parallel to the analytical and numerical work dealing with rotating flexible bladed-disc-shaft assemblies, efforts are made to extend experimental modal analysis techniques on rotating structures (Irretier and Reuter, 1995; 1997). Generally, this requires that the modal identification algorithms are based on time-variant systems, to account for anisotropic effects in the bladed-discs or shafts of the rotating assembly (Reuter, 1997; Irretier, 1998). In addition to the numerical procedure and results described in the paper, corresponding experimental results are presented which are based on this extended modal testing and identification technique.

\section{THEORETICAL BACKGROUND}

The method presented here has been developed in order to deal with turbomachinery wheels with complex shapes and geometry. The motion equations, expressed in a body fixed coordinate system, of a flexible bladed-disc mounted on a flexible shaft rotating at a given uniform angular velocity, can be expressed as:

$$
\begin{gathered}
{\left[\mathrm{KE}+\mathrm{KG}\left(\{\delta\}_{\mathrm{s}}\right)-\mathrm{KS}\right]\{\delta\}_{\mathrm{s}}=\left\{\mathrm{FC}\left(\Omega^{2}\right)\right\},} \\
{[\mathrm{M}]\{\ddot{\delta}\}_{\mathrm{d}}+[\mathrm{CM}+\mathrm{CR}]\{\dot{\delta}\}_{\mathrm{d}}} \\
+[\mathrm{KE}+\mathrm{KG}-\mathrm{KS}]\{\delta\}_{\mathrm{d}}=\{0\}
\end{gathered}
$$

where $[\mathrm{M}]$ is the mass matrix, $[\mathrm{CM}]$ the mechanical damping matrix, $[\mathrm{CR}]$ the gyroscopic matrix, [KE] the elastic stiffness matrix, $[\mathrm{KG}]$ the geometric stiffness matrix, $[\mathrm{KS}]$ the supplementary stiffness matrix and $\{\mathrm{FC}\}$ the nodal centrifugal force vector. $\{\delta\}_{\mathrm{s}}$ is the static equilibrium position of the structure under centrifugal loading and $\{\delta\}_{\mathrm{d}}$ is the small amplitude dynamic displacement around the static position. For a given rotation speed $\Omega$, the solution of the non-linear system (1) using a NewtonRaphson procedure gives the static displacement 
vector. The stiffness matrix is then known and the dynamic problem can be solved.

When dealing with bladed-disc-shaft assemblies, the size of the problem can be reduced if their rotationally periodic characteristics are taken into account. Such structures are constituted with $N$ identical jointed sectors. According to the wave propagation theory in periodic media, Thomas (1979), the dynamic displacement vector of the different sectors $p$ is related to the corresponding quantities of a reference sector by the following phase relations:

$$
\{\delta\}_{\mathrm{d}}^{p}=\left\{\delta_{n}^{\mathrm{c}}\right\} \cos (p-1) \beta_{n}+\left\{\delta_{n}^{\mathrm{s}}\right\} \sin (p-1) \beta_{n},
$$

where $\{\delta\}_{\mathrm{d}}^{p}$ is the vibrational displacement of sector $p,\left\{\delta_{n}^{\mathrm{c}}\right\},\left\{\delta_{n}^{\mathrm{s}}\right\}$ are generalized quantities associated with the basic sector and $\beta_{n}=2 \pi n / N$ is the phase difference between the displacement of two adjacent sectors. $N$ is the total number of sectors and $n$, Fourier order, takes the discrete values:

$$
\begin{array}{ll}
n=0,1, \ldots, N / 2, & \text { if } N \text { is even } \\
n=0,1, \ldots,(N-1) / 2, & \text { if } N \text { is odd. }
\end{array}
$$

Applying the wave propagation relations (3), the dynamic problem (2) is divided into small size systems associated with each of the possible phase angles $\beta_{n}$ :

$$
\begin{aligned}
& {\left[\mathrm{M}_{n}\right]\left\{\ddot{\delta}_{n}\right\}+\left[\mathrm{CM}_{n}+\mathrm{CR}_{n}\right]\left\{\dot{\delta}_{n}\right\}} \\
& \quad+\left[\mathrm{KE}_{n}+\mathrm{KG}_{n}-\mathrm{KS}_{n}\right]\left\{\delta_{n}\right\}=\{0\},
\end{aligned}
$$

where $\left\{\delta_{n}\right\}=\left\langle\delta_{n}^{\mathrm{c}}, \delta_{n}^{\mathrm{s}}\right\rangle^{\mathrm{t}}$. When dealing with isolated bladed-discs, damping and gyroscopic effects are usually neglected and (5) becomes a Hermitian system which is solved only once, for an operating speed. When dealing with bladed-disc-shaft assemblies, gyroscopic effects can no longer be neglected and the variations of frequencies with respect to the rotation speed are relatively large. Consequently, it becomes necessary to solve a different complex and non-Hermitian eigenvalue problem for each value of the rotation speed in the operating range. In this case, using only the traditional reductions (Craig and Bampton, 1968) leads to a lengthy procedure which is still not computationally efficient. In the following, we shall show that the analysis cost of bladed-disc-shaft assemblies can be considerably reduced, without appreciable loss of accuracy, provided the rotating mode shapes are written as a linear combination of the associated non-rotating mode shapes.

\section{REDUCED DYNAMIC PROBLEM}

Let us first consider the undamped system at rest $(\Omega=0)$. Thus (5) reduces to:

$$
\left[\mathrm{M}_{n}\right]\left\{\ddot{\delta}_{n}\right\}+\left[\mathrm{KE}_{n}\right]\left\{\delta_{n}\right\}=\{0\} .
$$

The solution of (6) is performed after a condensation based on the efficient Craig and Bampton (1968) substructuring method. This solution gives the frequencies and mode shapes of the nonrotating system (Jacquet-Richardet et al., 1996). All the mode shapes calculated are grouped into modal matrices $\left[\Psi_{n}\right]$, used for the reduction of the rotating system (5). Assuming that:

$$
\left\{\delta_{n}\right\}=\left[\Psi_{n}\right]\left\{\mathrm{q}_{n}\right\}
$$

system (5) becomes:

$$
\left[\mathrm{m}_{n}\right]\left\{\ddot{\mathrm{q}}_{n}\right\}+\left[\mathrm{c}_{n}\right]\left\{\dot{\mathrm{q}}_{n}\right\}+\left[\mathrm{k}_{n}\right]\left\{\mathrm{q}_{n}\right\}=\{0\}
$$

with:

$$
\begin{aligned}
{\left[\mathrm{m}_{n}\right] } & =\left[\Psi_{n}\right]^{\mathrm{t}}\left[\mathrm{M}_{n}\right]\left[\Psi_{n}\right], \\
{\left[\mathrm{c}_{n}\right] } & =\left[\Psi_{n}\right]^{\mathrm{t}}\left[\mathrm{CM}_{n}+\mathrm{CR}_{n}\right]\left[\Psi_{n}\right], \\
{\left[\mathrm{k}_{n}\right] } & =\left[\Psi_{n}\right]^{\mathrm{t}}\left[\mathrm{KE}_{n}+\mathrm{KG}_{n}-\mathrm{KS}_{n}\right]\left[\Psi_{n}\right] .
\end{aligned}
$$

The solution of (6) and (8) is performed for all the possible values of the phase parameter $\beta_{n}=2 \pi n / N$ given by (4). The frequencies of the rotating system are given directly. The corresponding mode shapes are obtained after applying the two successive 
transformations, (7) and (3), to the calculated eigenvectors. The modes of the shaft can occur only with $n=0$ or $n=1$ ( $n=0$ torsion and longitudinal modes, $n=1$ bending modes). The modes of bladed-discs are always classified using an analogy with axisymmetric modes, which are mainly characterised by nodal lines lying along the diameters of the structure and having a constant angular spacing. They are either zero $(n=0)$, one $(n=1)$, two $(n=2)$ or more $(n>2)$ nodal diameter bending or torsion modes. Inertial disc-shaft coupling effects are important for modes associated with $n=0$ and $n=1$, because these modes are the only ones where a shaft motion can occur. The disc modes with zero-nodal-diameter, which are characterised by a resultant axial force, interact with the longitudinal shaft deformations. The modes with one-nodal-diameter, which exert a net pitching moment and a shearing force, interact with the shaft bending modes.

In order to be able to identify possible resonance points, the frequencies are calculated for the whole operating range of the structure and the results classically reported on a Campbell diagram, which illustrates the dependence of the natural frequencies on the rotor speed $\Omega$. When using the proposed analysis method, the non-rotating mode shapes of the assembly are calculated only once. Then, the following steps are involved for each rotation speed considered:

- the static problem is solved;

- the dynamic problem (5) is reduced according to (7);

- the reduced system (8) is solved.

Compared to traditional procedures, the computer cost saving is considerable and allows precise analyses of complex industrial structures using efficient workstations.

\section{APPLICATION}

For the verification of the numerical model and analysis and to study coupling effects of an elastic

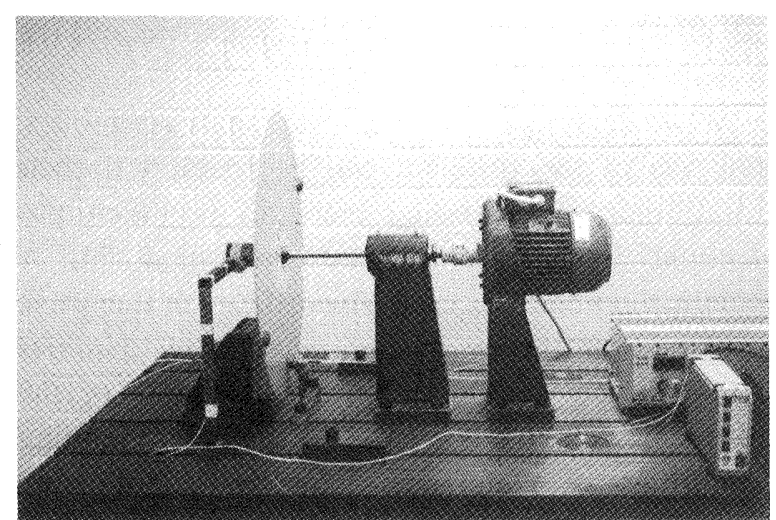

FIGURE 1 Elastic disc on a flexible shaft.

disc on a flexible shaft under rotation, a test stand was designed as shown in Fig. 1 (Reuter, 1997). It consists of a thin circular steel disc (outer radius $300 \mathrm{~mm}$, thickness $3 \mathrm{~mm}$ ) fixed in an overhung position on an elastic circular steel shaft (length $175 \mathrm{~mm}$, diameter $15 \mathrm{~mm}$ ). The supported shaft prolongation (length $101.5 \mathrm{~mm}$, diameter $28 \mathrm{~mm}$ ) in two ball bearings can be considered to be rigid in comparison to the flexibility of the overhung shaft. Two different types of disc tuning states were investigated: (I) Completely tuned and (II) mistuned by two diametral masses (each $0.047 \mathrm{~kg}$ at a radius of $280 \mathrm{~mm}$ as visible in Fig. 1). For systems (I) and (II) numerical calculations were carried out at INSA Lyon by the technique described above while for system (II) additional experimental investigations were carried out at the University of Kassel. For these tests, a step sine excitation by a magnetic exciter with an integrated force transducer was used (Fig. 2) and the response was measured by an eddy current pick-up, both without contact with the rotating disc. Both are visible in Fig. 1, too, and the schematic representation of the test stand is given in Fig. 3 (Irretier and Reuter, 1995). From the excitation and response signals, frequency response functions were determined for the rotating system based on a theory for timevariant systems and, by curve fitting algorithms in the frequency domain. The modal parameters were identified with respect to the stationary frame (index s, Fig. 3). If desired, they are converted to 


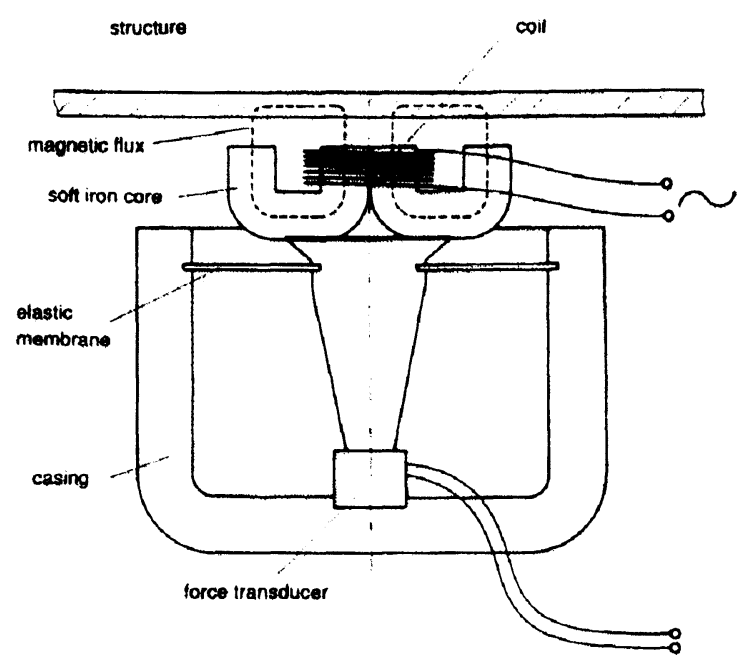

FIGURE 2 Magnetic exciter with piezo-electric force transducer. other reference frames, e.g., the rotating frame (Reuter, 1997)

Considering the numerical model, a portion of $54^{\circ}$ of the disk-shaft assembly is meshed using 108 isoparametric brick finite elements with 20 nodes. The resulting mesh, presented in Fig. 4 (dark zone) comprises about 550 finite element nodes and, consequently, about 1650 degrees of freedom. The matrices associated with the disk elements are evaluated using a reduced Gaussian integration to avoid numerical shear locking. Damping effects are neglected. The natural frequencies associated with the first modes calculated are conveyed onto the Campbell diagram presented in Fig. 5. At rest, these modes can be classified as follows. The first mode is mainly a shaft first bending mode, influenced by the disk mode with one-nodal-diameter. The second mode is a disk mode without any nodal diameter or nodal circle (umbrella mode). The third mode is a

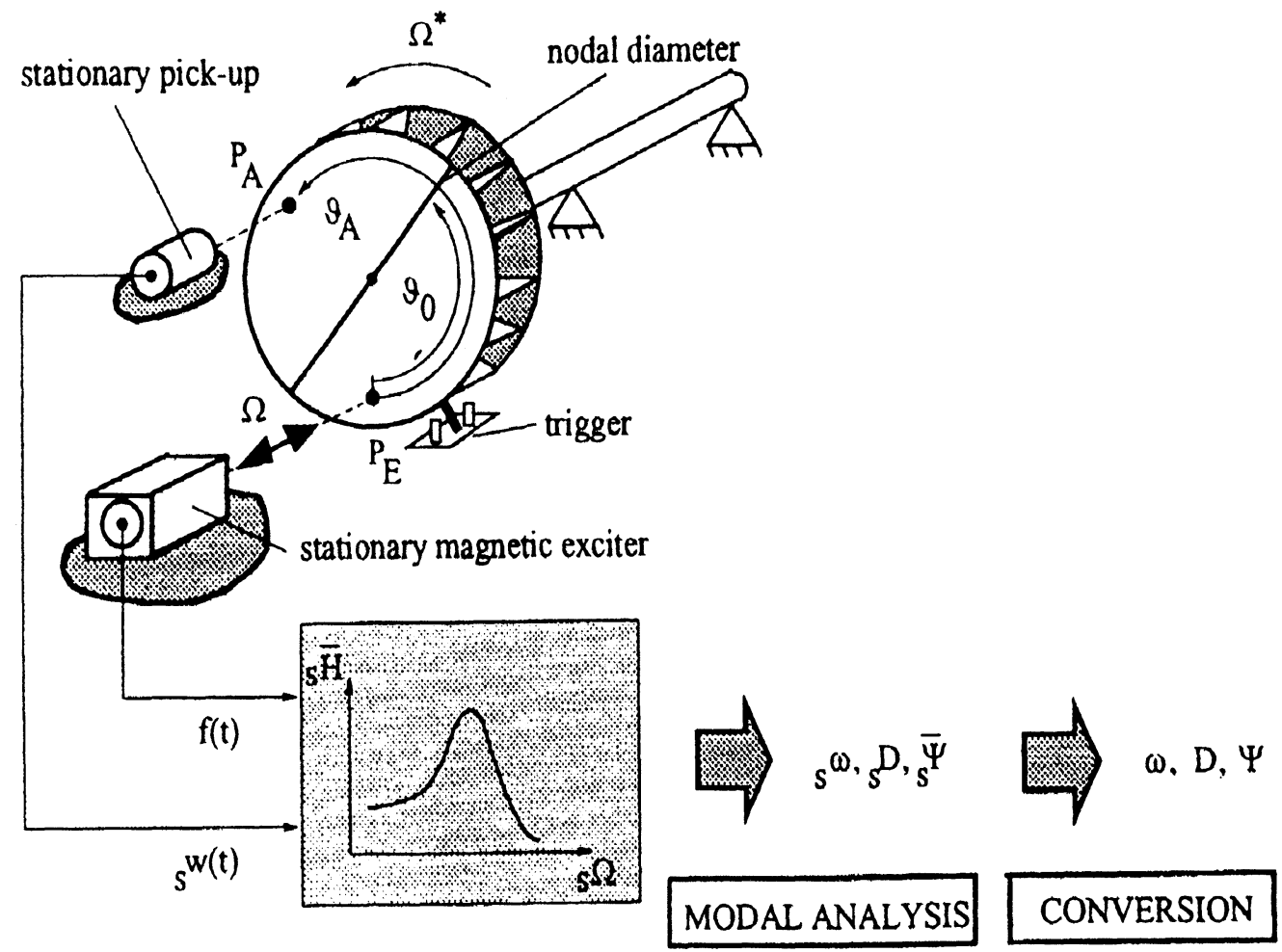

FIGURE 3 Experimental modal analysis of rotating structures. 


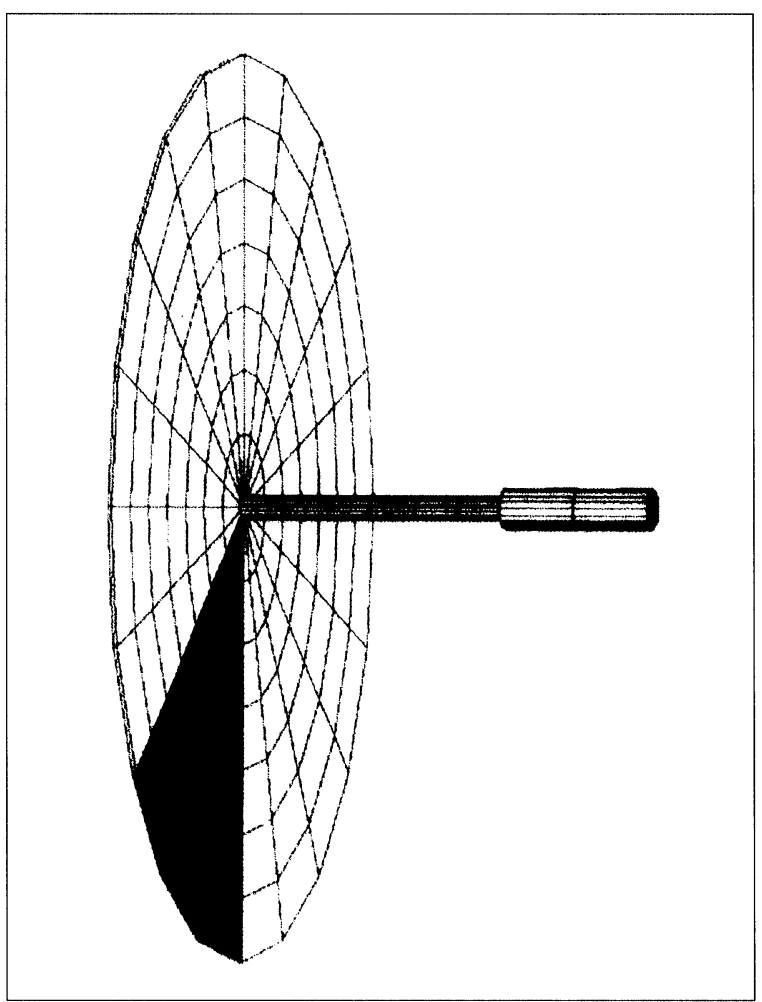

FIGURE 4 Finite element mesh of the assembly.

shaft bending mode strongly coupled with the onenodal-diameter disk mode. Finally, the fourth mode is a disk mode with two nodal diameters. The progression of the disk modes with rotation is only induced by centrifugal effects (stress stiffening and spin softening). As predicted, no coupling occurs between these modes and shaft bending. When rotating, each shaft mode splits into backward and forward branches, due to gyroscopic effects. When the speed increases, there is a clear coupling between the first and second shaft bending modes.

\section{Influence of Disk and Shaft Flexibility}

The influence of disk flexibility on the overall behavior is illustrated by comparison of the results presented in Fig. 5, with the results obtained using a rotordynamic approach (rigid disk). The comparison, which naturally involves only shaft modes, is

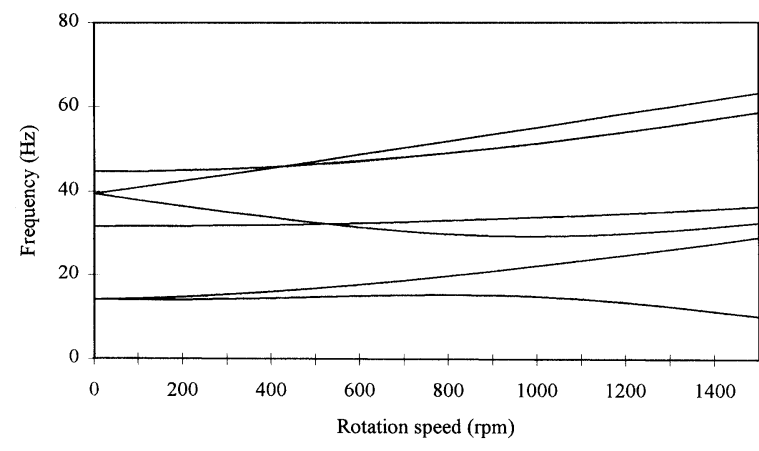

FIGURE 5 Campbell diagram (rotating frame).

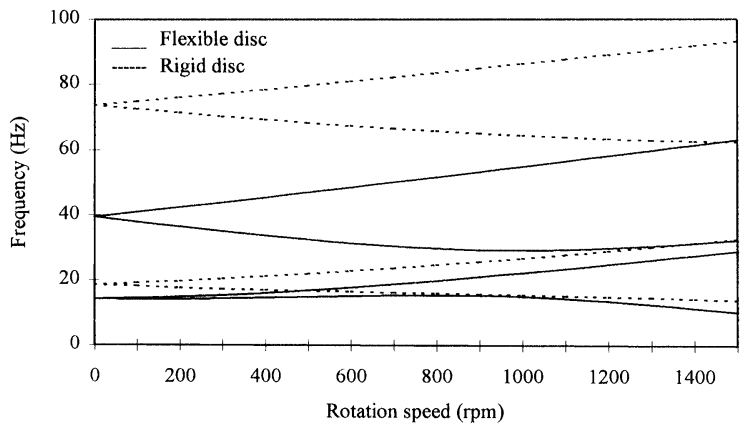

FIGURE 6 Influence of disc flexibility.

presented in Fig. 6. When examining Fig. 6, it appears clearly that the influence of disk flexibility is considerable here. At rest, the first frequency given by the rotordynamic approach is overestimated by a factor of $31 \%$ and the second one is overestimated by a factor of $87 \%$. This last result illustrates the coupled nature of the second shaft bending mode with the one-nodal-diameter disk mode. Considering the evolution of frequencies with rotation, the forward branches (lower branches in the rotating frame) are much more influenced by disk flexibility effects than the backward branches.

The influence of shaft flexibility is illustrated in Fig. 7, where the flexible disc-shaft assembly results (solid lines) are compared with results associated with a rigid shaft model (dotted lines). The rigid shaft results were obtained by considering the disk isolated and clamped at its inner diameter. 
Figure 7 confirms that the zero- and two-nodaldiameter disk modes, identified previously, are not influenced by shaft bending. On the contrary, the highly coupled nature of shaft bending and one-nodal-diameter disk bending is here also highlighted. For the flexible assembly, the one-nodaldiameter mode has been identified as a component of the first and third modes: at rest $\mathrm{f} 1 \doteq 14.2 \mathrm{~Hz}$ and $\mathrm{f} 3=39.4 \mathrm{~Hz}$. The pure one-nodal-diameter disk mode is just between these two frequencies: $\mathrm{f}=21.6 \mathrm{~Hz}$. When the speed increases, it can be noticed that the backward branch of the first mode and the forward branch of the third mode of the flexible assembly, evolve toward a pure one-nodaldiameter disk mode.

\section{Influence of Mistuning}

The influence of mistuning has been tested by adding two diametrically opposed point masses of

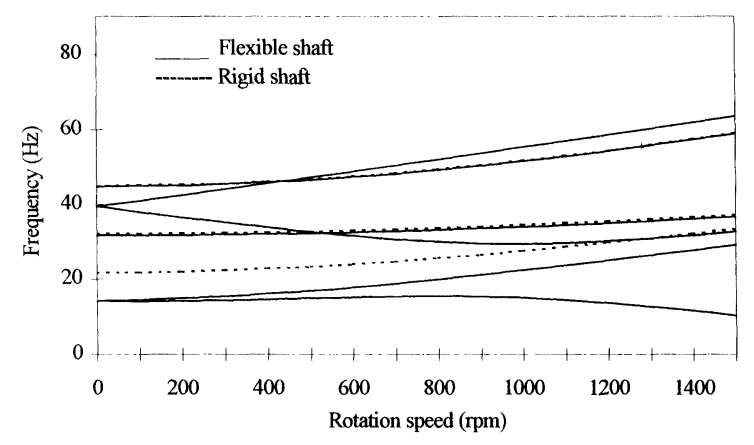

FIGURE 7 Influence of shaft flexibility. $m=0.047 \mathrm{~kg}$ each, on the disk, at a radius $R=280 \mathrm{~mm}$. Due to these two masses the assembly is no longer axisymmetric and, consequently, the choice of the basic cyclic sector is no longer free but should take up half of the structure. The resulting mesh, based on the same element size as previously, comprises 360 finite elements and 2200 nodes. In this case, the computational effort needed is obviously much more considerable.

Frequency splitting can be noticed due to mistuning. As the mistuning introduced in the system is relatively low, Table I shows that the splitting induced is not very pronounced. For comparison, the test results are also presented in Table I (with the exception of mode 3 which was not investigated in the tests). The experimental frequencies for the first shaft bending mode $(n=1)$ are almost $6 \%$ higher than the numerical results. The main reason for this discrepancy is the slightly longer overhung shaft considered in the numerical calculations ( $180 \mathrm{~mm}$ instead of $175 \mathrm{~mm}$ ). For the elastic disc mode frequencies with $n=0$ and $n=2$, the difference between the test and numerical results is much less, being around $1 \%$. The splitting tendency due to mistuning is very well predicted for the $n=1$ and $n=2$ diameter mode as can be seen in Table I.

The Campbell diagram for the mistuned assembly is presented in Fig. 8 for the modes under consideration. Besides the curves representing the numerical results, test results are also shown by included points. Again, the agreement between

TABLE I Frequencies at rest, influence of mistuning

\begin{tabular}{|c|c|c|c|c|c|}
\hline \multirow[t]{2}{*}{ Mode $(n)$} & \multicolumn{3}{|c|}{ Numerical results } & \multicolumn{2}{|c|}{ Test results } \\
\hline & $\begin{array}{l}\text { Tuned assembly } \\
F(\mathrm{~Hz}) \Omega=0 \mathrm{rpm}\end{array}$ & $\begin{array}{l}\text { Mistuned assembly } \\
F(\mathrm{~Hz}) \Omega=0 \mathrm{rpm}\end{array}$ & $\Delta(\%)$ & $\begin{array}{l}\text { Mistuned assembly } \\
F(\mathrm{~Hz}) \Omega=0 \mathrm{rpm}\end{array}$ & $\Delta(\%)$ \\
\hline \multirow[t]{2}{*}{$1(1)$} & 14.1 & 13.8 & -2.1 & 14.5 & 5.1 \\
\hline & 14.1 & 14.1 & 0.0 & 15.0 & 6.4 \\
\hline \multirow[t]{2}{*}{$2(0)$} & 31.6 & 31.1 & -1.6 & 31.5 & 1.3 \\
\hline & 31.6 & 31.1 & -1.6 & 31.5 & 1.3 \\
\hline \multirow[t]{2}{*}{$3(1)$} & 39.4 & 39.0 & -1.0 & - & - \\
\hline & 39.4 & 39.1 & -0.8 & - & - \\
\hline \multirow[t]{2}{*}{$4(2)$} & 44.7 & 43.4 & -2.9 & 43.8 & 0.9 \\
\hline & 44.7 & 44.7 & 0.0 & 44.9 & 0.4 \\
\hline
\end{tabular}




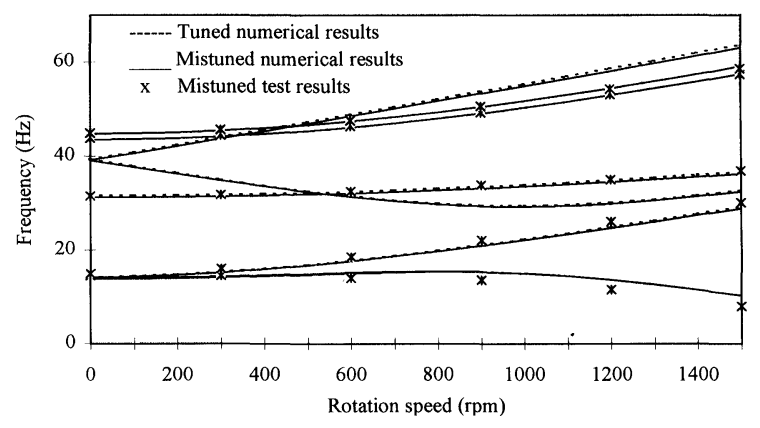

FIGURE 8 Mistuned assembly.

both results is excellent with slightly higher deviations for the first shaft bending mode, as already indicated earlier for zero rotation.

\section{CONCLUSION}

A numerical technique was presented for the calculation of natural frequencies and mode shapes of coupled elastic shaft-disc systems under rotation. It is based on a finite element modal approach combined with the wave propagation method for cyclic symmetric structures and a component mode reduction technique, both to reduce the required numerical effort considerably. Confirmed by related experimental results for a rotating mistuned disc on a flexible overhung shaft, the proposed numerical method proves to be a powerful technique for the frequency analysis of these types of rotating structures. The results show that the one-nodal-diameter modes of the disc are highly coupled with the shaft bending modes for the test example considered. For these coupled modes, the influence of rotation on the natural frequencies becomes apparent by both the centrifugal stiffening as well as the gyroscopic effect. As expected, the umbrella mode and the two-nodaldiameter mode of the disc which are not coupled with the shaft flexibility are only influenced by the centrifugal stiffening effect.

\section{Acknowledgment}

This paper is dedicated to the late Prof. Dr. R. Henry with whom the first author became a colleague and friend in 1987. The co-operation started then gave rise to a long lasting relationship between the Institute of Mechanics, University of Kassel and the Laboratoire de Mécanique des Structures, INSA de Lyon. This joint paper is an expression of this relationship which the authors hope to continue in the future.

\section{References}

Brémand, P., Ferraris, G. and Lalanne, M., 1986, Prediction of natural frequencies of flexible shaft-disc system, The Shock and Vibration Bulletin 56(2-3), 71-80.

Chivers, D.R. and Nelson, H.D., 1975, The natural frequencies and critical speeds of a rotating flexible shaft-disc system, J. of Engineering for Industry 97, 881-886.

Chun, S.B. and Lee, C.W., 1996, Vibration analysis of shaftbladed disk systems by using substructure synthesis and assumed modes method, J. of Sound and Vib. 189(5), 587-608.

Craig, R.R. and Bampton, M.C.C., 1968, Coupling of substructures for dynamic analysis, AJAA J. 6, 1313-1319.

Crawley, E.F., 1983, In plane inertial coupling in tuned and severely mistuned bladed disks, J. of Engineering for Power 105, 585-590

Crawley, E.F., Ducharme, E.H. and Mokadam, D.R., 1986, Analytical and experimental investigation of the coupled bladed disk/shaft whirl of a cantilever turbofan, $J$. of Engineering for Gas Turbine and Power 108, 567-576.

Dopkin, J.A. and Shoup, T.E., 1974, Rotor resonant speed reduction caused by flexibility of disks, $J$. of Engineering for Industry 96(4), 1328-1333.

Géradin, M. and Kill, N., 1984, A new approach to finite element modeling of flexible rotors, Engineering Computations 1, 52-64.

Géradin, M. and Kill, N., 1986, A three dimensional approach to dynamic analysis of rotating shaft disc flexible systems, Proc. 2nd Int. Conf. on Rotordynamics IFToMM, Tokyo, pp. $87-93$.

Henry, R. and Ferraris, G., 1984, Substructuring and wave propagation: an efficient technique for impeller dynamic analysis, J. of Eng. for Gas Turbine and Power 106(1), 2-10.

Hohlrieder, M. 1994, Zur statischen und dynamischen analyse rotierender elastischer strukturen bei transienten betriebsbedingungen, Dissertation, Fachbereich Maschinenbau, Universität Gh Kassel.

Hohlrieder, M. and Irretier, H., 1997, Comparison of the rotor dynamic and structure dynamic calculation of compressors, J. of Engineering for Gas Turbine and Power 119(2), 425-429.

Irretier, H. and Reuter, F., 1995, Experimental modal analysis of rotating disk systems, Proc. 15th ASME Conference on Mechanical Vibration and Noise, Boston, pp. 1201-1206

Irretier, H. and Reuter, F., 1997, Frequency response functions of rotating time-varying systems. Proc. 15th Int. Modal Analysis Conference, Orlando, pp. 350-356.

Irretier, H., 1998, Mathematical foundations of experimental modal analysis in rotor dynamics, to appear in $J$. of Mechanical Systems and Signal Processing.

Jacquet-Richardet, G., Ferraris, G. and Rieutord, P., 1996, Frequencies and modes of rotating flexible bladed disc-shaft assemblies: a global cyclic symmetry approach, J. of Sound and Vibration 191(5), 901-915. 
Khader, N. and Loewy, R.G., 1990, Shaft flexibility effects on the forced response of a bladed disk assembly, $J$. of Sound and Vibration 139(3), 469-485.

Khader, N. and Masoud, S., 1991, Vibration of mistuned bladed disks supported by flexible continuous shafts, $J$. of Sound and Vibration 149(3), $471-488$

Klompas, N., 1978, Significance of disk flexing in viscous damped jet engine dynamics, $J$. of Engineering for Power 100, 647-654.

Loewy, R.G. and Khader, N., 1984, Structural dynamics of rotating bladed disk assemblies coupled with flexible shaft motions, AIAA J. 22(9), 1319-1327.

Palladino, J.A. and Rossettos, J.N., 1982, Finite element analysis of the dynamics of flexible disk rotor systems, ASME Paper $82 \mathrm{GT} 240$.

Reuter, F., 1997, Zur modalen Theorie periodisch zeitvarianter systeme und ihrer experimentellen umsetzung auf rotierende, scheibenförmige strukturen, Dissertation, Fachbereich Maschinenbau, Universität Gh Kassel.

Stephenson, R.W. and Rouch, K.E., 1993, Modeling rotating shafts using axisymmetric solid finite elements with matrix reduction, $J$. of Vibration and Acoustics 115, 484-489.

Thomas, D.L., 1979, Dynamics of rotationally periodic structures, Int. J. for Num. Meth. in Eng. 14, 81-102.

Wu, F. and Flowers, C.T., 1992, A transfer matrix technique for evaluating the natural frequencies and critical speeds of a rotor with multiple flexible disks, J. of Vibration and Acoustics 114 $242-248$.

Zhang, W., Wang, W., Wang, H. and Tang, J., 1994, Analytical and experimental investigation of bladed-disk/shaft coupled system, Proc. 4th Int. Conf. on Rotordynamics IFToMM, Chicago, pp. 63-68. 


\section{ait \\ ENERGY MATERIALS}

M A N E Y publishing

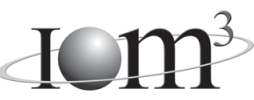

\section{Materials Science \& Engineering for Energy Systems}

Maney Publishing on behalf of the Institute of Materials, Minerals and Mining

The Institute of Materials, Minerals \& Mining

Economic and environmental factors are creating ever greater pressures for the efficient generation, transmission and use of energy. Materials developments are crucial to progress in all these areas: to innovation in design; to extending lifetime and maintenance intervals; and to successful operation in more demanding environments. Drawing together the broad community with interests in these areas, Energy Materials addresses materials needs in future energy generation, transmission, utilisation, conservation and storage. The journal covers thermal generation and gas turbines; renewable power (wind, wave, tidal, hydro, solar and geothermal); fuel cells (low and high temperature); materials issues relevant to biomass and biotechnology; nuclear power generation (fission and fusion); hydrogen generation and storage in the context of the 'hydrogen economy'; and the transmission and storage of the energy produced.

As well as publishing high-quality peer-reviewed research, Energy Materials promotes discussion of issues common to all sectors, through commissioned reviews and commentaries. The journal includes coverage of energy economics and policy, and broader social issues, since the political and legislative context influence research and investment decisions.

\section{CALL FOR PAPERS}

Contributions to the journal should be submitted online at http://ema.edmgr.com

To view the Notes for Contributors please visit: www.maney.co.uk/journals/notes/ema

Upon publication in 2006, this journal will be available via the Ingenta Connect journals service. To view free sample content online visit: www.ingentaconnect.com/content/maney

For further information please contact:

Maney Publishing UK

Tel: +44 (0)113 2497481 Fax: +44 (0)1132486983 Email: subscriptions@maney.co.uk

or

Maney Publishing North America

Tel (toll free): 8662975154 Fax: 6173546875 Email: maney@maneyusa.com

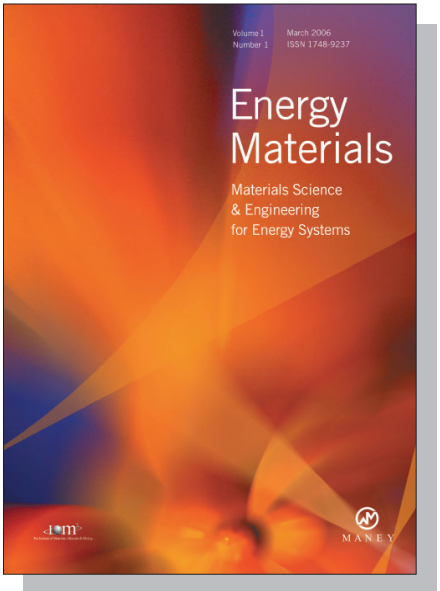

EDITORS

Dr Fujio Abe

NIMS, Japan

Dr John Hald, IPL-MPT, Technical University of Denmark, Denmark

Dr R Viswanathan, EPRI, USA

\section{SUBSCRIPTION INFORMATION}

Volume 1 (2006), 4 issues per year

Print ISSN: 1748-9237 Online ISSN: 1748-9245

Individual rate: $£ 76.00 / U S \$ 141.00$

Institutional rate: $£ 235.00 /$ US $\$ 435.00$

Online-only institutional rate: $£ 199.00 / U S \$ 367.00$

For special $\mathrm{IOM}^{3}$ member rates please email

subscriptions@maney.co.uk

\section{For further information or to subscribe online please visit www.maney.co.uk}



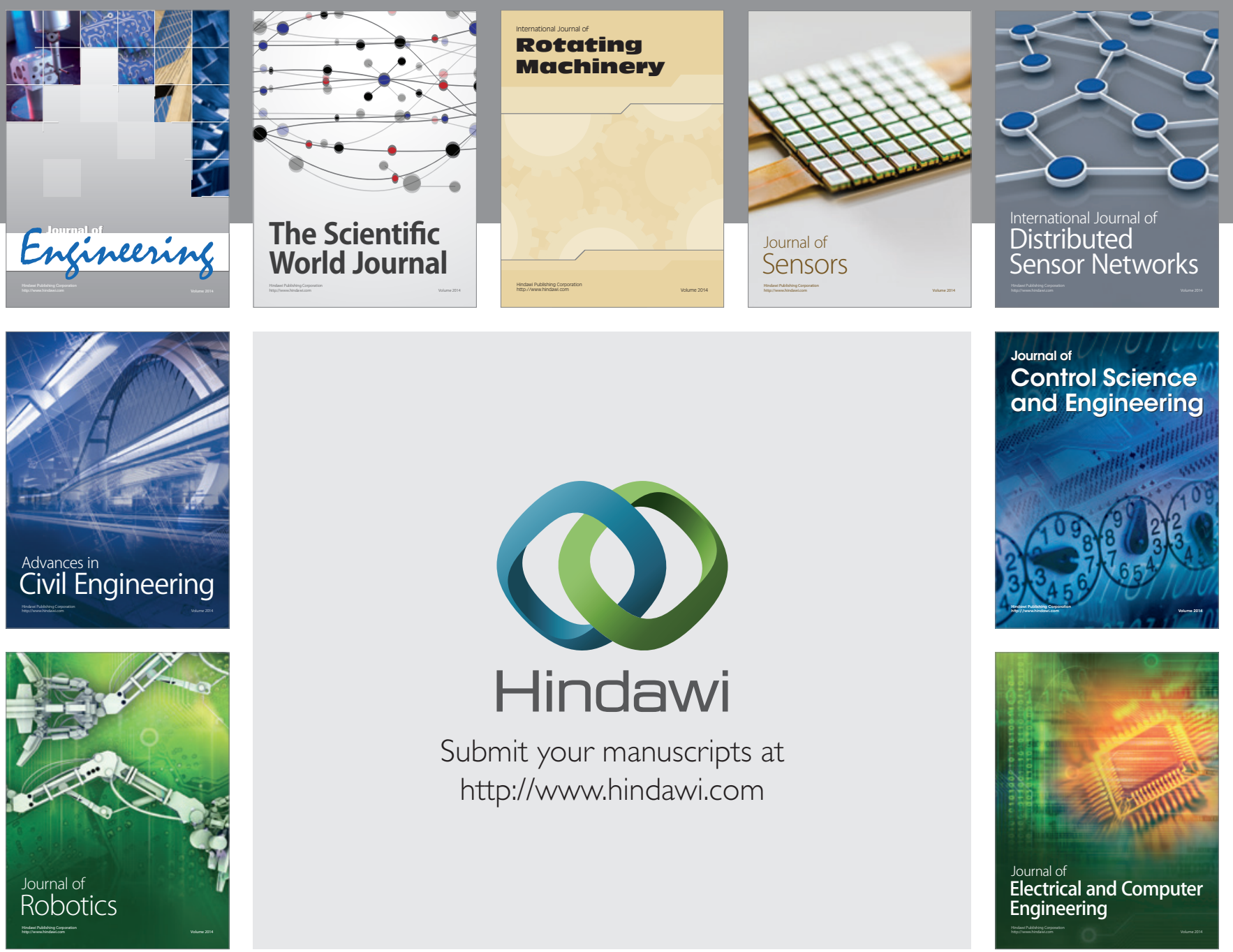

Submit your manuscripts at

http://www.hindawi.com
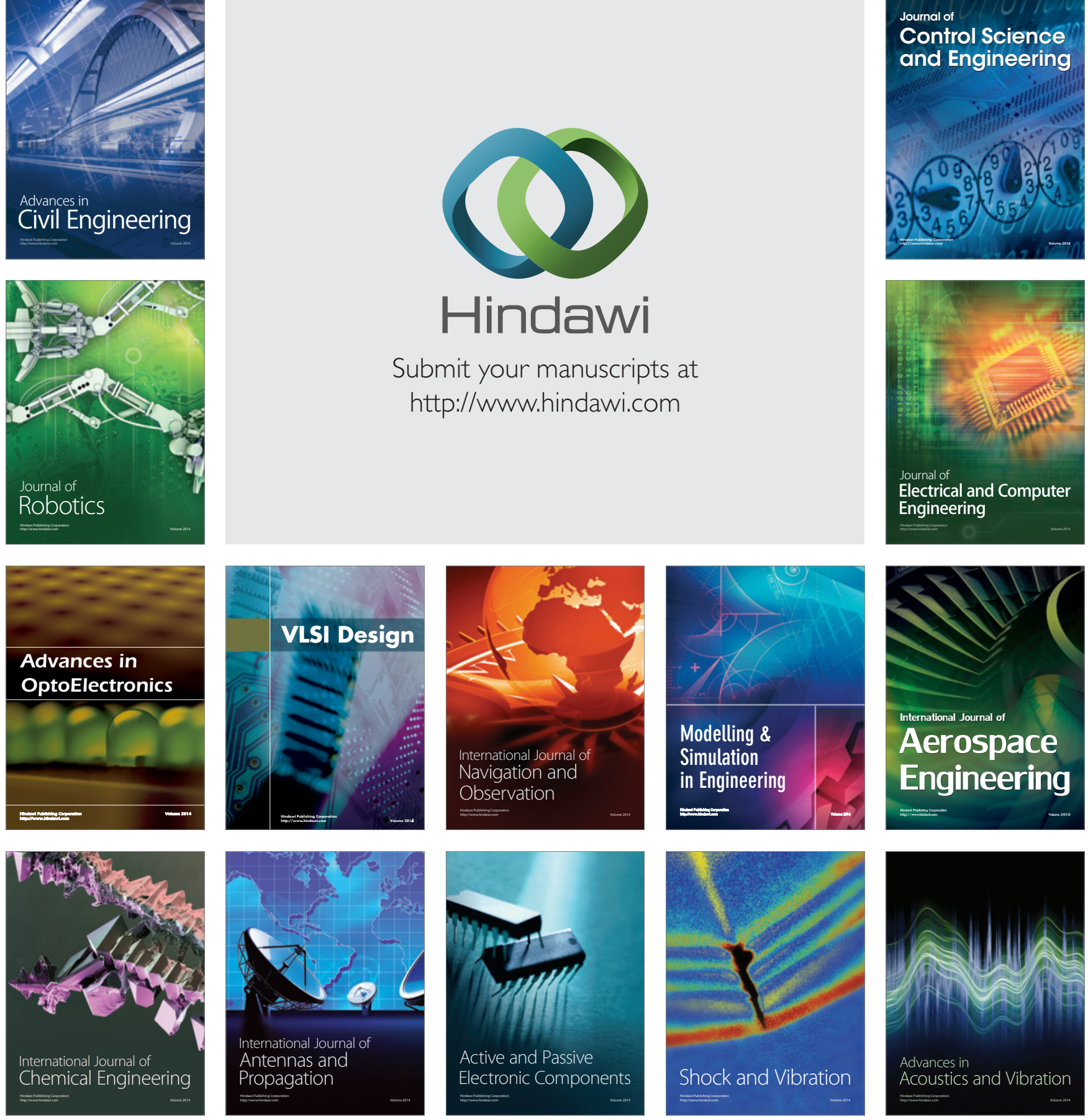